\title{
Glaucocalyxin B induces apoptosis and autophagy in human cervical cancer cells
}

\author{
YING PAN*, JIEYU BAI*, FANGFANG SHEN, LI SUN, QUANZHONG HE and BING SU \\ Xinxiang Key Lab of Translational Cancer Research, The Third Affiliated Hospital of Xinxiang Medical University, \\ Xinxiang, Henan 453003, P.R. China
}

Received February 6, 2016; Accepted June 3, 2016

DOI: $10.3892 / \mathrm{mmr} .2016 .5450$

\begin{abstract}
Glaucocalyxin (Gln), an ent-kaurane diterpenoid isolated from the Chinese traditional medicine, Rabdosia japonica, represents a novel class of anticancer drugs. GlnA is one of the three major forms of Gln and has demonstrated potent anticancer effects in a variety of cancer types. GlnB has only one structural difference from GlnA, an acetylated hydroxyl group at C14. This acetyl group results in high liposolubility and may enhance the antitumor activity of ent-kaurane diterpenoid GlnB. However, few studies have reported the role of GlnB in cancer. The present study investigated the effect of GlnB in cervical cancer proliferation and cell death. Treatment with GlnB inhibits the proliferation of $\mathrm{HeLa}$ and $\mathrm{SiHa}$ cervical cancer cell lines in a dose-dependent manner, as assessed by 3-(4,5-dimethylthiazol-2-yl)-2,5 diphenyl tetrazolium bromide assays. In addition, GlnB increases the apoptotic cell population of $\mathrm{HeLa}$ and $\mathrm{SiHa}$ cells, as determined by fluorescence-activated cell sorting analysis and enhanced poly (ADP-ribose) polymerase 1 cleavage by western blotting. GlnB also induces increased light chain 3 II/I protein cleavage in both cells, indicating the induction of autophagy. Furthermore, GlnB treatment increased the expression of phosphatase and tensin homolog and decreased the expression of phosphorylated-protein kinase B (Akt) in HeLa and $\mathrm{SiHa}$ cells, as assessed by western blotting. Taken together, the present results demonstrated that GlnB inhibited the proliferation of human cervical cancer cells in vitro through the induction of apoptosis and autophagy, which may be mediated
\end{abstract}

Correspondence to: Dr Ying Pan or Dr Bing Su, Xinxiang Key Lab of Translational Cancer Research, The Third Affiliated Hospital of Xinxiang Medical University, 6 Hualan Avenue, Xinxiang, Henan 453003, P.R. China

E-mail: panying@xxmu.edu.cn

E-mail: su_bing2000@hotmail.com

*Contributed equally

Key words: glaucocalyxin B, cervical cancer, Chinese traditional medicine, apoptosis, autophagy by the phosphatidylinositol-4,5-bisphosphate 3-kinase/Akt signaling pathway.

\section{Introduction}

Cervical cancer is the most common malignant tumor in the female reproductive tract. Previously, increased incidence and mortality of cervical cancer, and a younger age at diagnosis were reported, requiring further research and more effective treatment of this deadly disease (1).

Currently, the combination of radiotherapy with platinum-based chemotherapy is the gold-standard treatment for advanced cervical cancer (2). Although the addition of platinum-based chemotherapy to radiotherapy has increased the 5-year survival of advanced-stage cervical cancer patients (3), systemic toxicity limits the use of high-dose chemotherapeutic drugs.

Therefore, alternative therapies for cervical cancer are urgently required. Complementary and alternative medicines can perhaps benefit patients with cervical cancer as an adjunctive therapy. Among them, Chinese traditional medicine has become increasingly prominent and popular in cancer patients due to its efficacy and low toxicity (4).

Glaucocalyxin (Gln), an ent-kaurane diterpenoid isolated from the Chinese traditional medicine, Rabdosia japonica, has been used in traditional medicine as an antibacterial, anti-inflammatory and anticancer agent (5). Gln has three major forms, GlnA, GlnB and GlnC. Previously, GlnA has been intensively studied for its potent anticancer effects and its diverse molecular targets involved in tumorigenesis (6-8).

By contrast, few studies have investigated the functions of GlnB. GlnB has only one structural difference from GlnA, an acetylated hydroxyl group at $\mathrm{C} 14$. This acetyl group results in high liposolubility and may enhance the antitumor activity of ent-kaurane diterpenoid. The role of GlnB in cancer remains largely unknown. The present study reported that GlnB exerts its anticancer effects by inducing apoptosis and autophagy, and inhibiting proliferation in cervical cancer cells.

\section{Materials and methods}

Cell culture. Human HeLa and SiHa cervical cancer cells were obtained from the Cell Bank of Type Culture Collection of Chinese Academy of Sciences (Shanghai, China). The cell lines 
were maintained in modified Eagle's media, containing $10 \%$ fetal bovine serum (FBS) at $37^{\circ} \mathrm{C}$ in a $5 \% \mathrm{CO}_{2}$ atmosphere.

Antibodies and reagents. The following antibodies were used in the present study: Rabbit anti-light chain (LC)3 (cat. no. 14600-1-AP), rabbit anti-poly (ADP-ribose) polymerase 1 (PARP1; cat. no. 13371-1-AP) and anti-glyceraldehyde 3-phosphate dehydrogenase (cat. no. 10494-1-AP; ProteinTech, Rosemont, IL, USA); rabbit anti-phosphorylated protein kinase B (p-)AKT ${ }^{\mathrm{S} 433}$ (cat. no. 9271) and rabbit AKT (cat. no. 9272) (Cell Signaling Technology, Inc., Beverly, MA, USA) (Cell Signaling Technology, Inc., Beverly, MA, USA); and rabbit anti-phosphatase and tensin homolog (PTEN; cat. no. ab31392 Abcam, Cambridge, MA, USA). The annexin V-fluorescent isothiocyanate Apoptosis Detection kit was purchased from Beyotime Institute of Biotechnology (Jiangsu, China).

Chemicals. GlnB, kindly provided by Dr Suping Bai (School of Pharmacy, Xinxiang Medical University, Xinxiang, China) was dissolved in dimethylsulfoxide (DMSO) to make $64 \mathrm{mmol} / \mathrm{l}$ stock solutions that was used for all experiments in the present study. Both 3-(4,5-dimethylthiazol-2-yl)-2,5-diphenyltetrazolium bromide (MTT) and DMSO were purchased from MP Biomedical, LLC (Santa Ana, CA, USA).

MTT assay. The cells were incubated overnight at $37^{\circ} \mathrm{C}$ in $5 \% \mathrm{CO}_{2}$ in media containing $10 \% \mathrm{FBS}$ at a concentration of 1,000 cells/well of a 96-well plate. On the next day, the cells were treated with either vehicle control (DMSO) or varying concentrations of $\mathrm{GlnB}$, and were allowed to grow for an additional $72 \mathrm{~h}$. Cell proliferation was assessed using the MTT assay. Following the addition of MTT (20 $\mu 1$ of $5 \mathrm{mg} / \mathrm{ml}$ MTT/well), the plate was incubated at $37^{\circ} \mathrm{C}$ for $4-5 \mathrm{~h}$. The media was subsequently removed and $150 \mu 1$ DMSO was added. The plate was then incubated in the same conditions for $5 \mathrm{~min}$. Proliferation was quantified using a plate reader at an optical density (OD) of $570 \mathrm{~nm}$. The cell growth inhibition was calculated as $\left(\mathrm{OD}_{\text {test }}-\mathrm{OD}_{0 \mathrm{~h}}\right) /\left(\mathrm{OD}_{\text {control }}-\mathrm{OD}_{0 \mathrm{~h}}\right) \times 10$ $0 \%$. A cell growth inhibition curve was generated by plotting cell growth inhibition against drug concentration, and the half-maximal inhibitory concentration $\left(\mathrm{IC}_{50}\right)$ was determined using GraphPad Prism 5 software (GraphPad Software, Inc., La Jolla, CA, USA).

Apoptosis assay. Apoptosis was analyzed using annexin V/propidium iodide (PI) staining, according to the manufacturer's protocol. Briefly, the cells were seeded into $60-\mathrm{mm}$ dishes. On the next day, the cells were treated with either DMSO or varying concentrations of GlnB. After $24 \mathrm{~h}$, the cells were collected by trypsinization, washed twice with cold phosphate-buffered saline and were subsequently resuspended in $1 \mathrm{X}$ binding buffer at a concentration of $1 \times 10^{6} \mathrm{cells} / \mathrm{ml}$. A total of $100 \mu \mathrm{l}$ of the solution was transferred to a $5 \mathrm{ml}$ culture tube, followed by the addition of $400 \mu 11 \mathrm{X}$ binding buffer, and stained with annexin $\mathrm{V}$ and PI. The stained cells were immediately analyzed on a FACScan flow cytometer. The stained cells were immediately collected using a BD FACScan flow cytometer (BD, Franklin Lakes, NJ, USA), and data were analyzed using the Winlist program 8.0 (Verity House, Topsham, ME, USA).
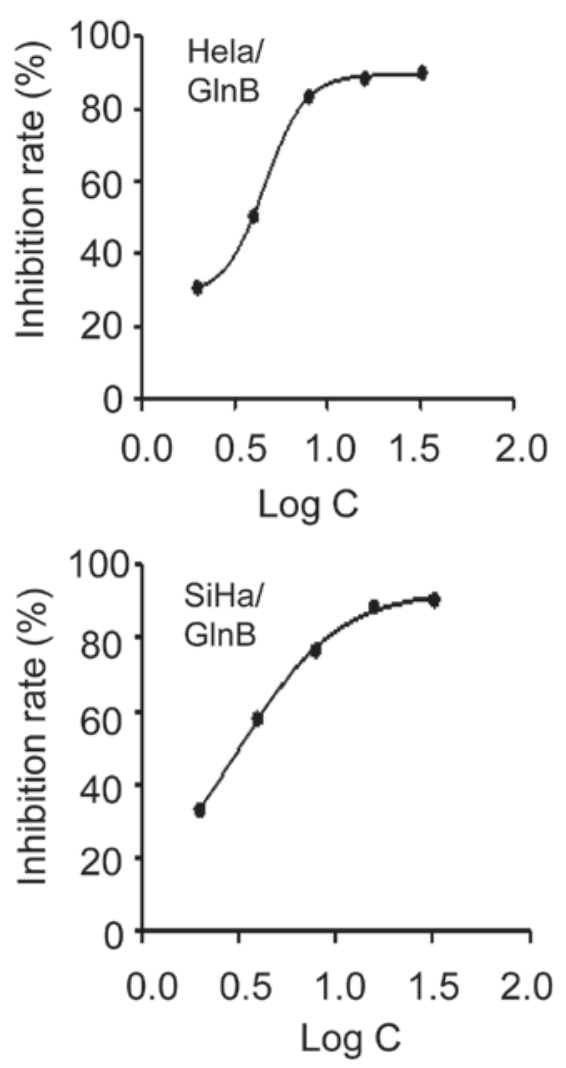

Figure 1. GlnB inhibits the proliferation of HeLa and SiHa cervical cancer cell lines. HeLa and $\mathrm{SiHa}$ cells were treated with various concentrations of GlnB $(2,4,8,16$, and $32 \mu \mathrm{M})$ for $72 \mathrm{~h}$. Cell viability was measured using the 3-(4,5-dimethylthiazol-2-yl)-2,5 diphenyl tetrazolium bromide assay. The $\mathrm{IC}_{50}$ of GlnB for HeLa and SiHa cells was 4.61 and $3.11 \mu \mathrm{mol} / 1$, respectively. GlnB, glaucocalyxin B; Log C, compound concentration.

Western blotting. Cells grown in the presence or absence of GlnB were lysed in radioimmunoprecipitation buffer. A total of $40 \mu \mathrm{g}$ total proteins per sample was separated by sodium dodecyl sulfate-polyacrylamide gel electrophoresis. The proteins were subsequently blotted onto polyvinylidene difluoride membranes and were blocked for $1 \mathrm{~h}$ at room temperature with $5 \%$ bovine serum albumen (BSA) in $1 \mathrm{X}$ Tris-buffered saline containing 0.1\% Tween-20 (TBST). Following blocking, the membranes were probed with anti-PARP1 (1:1,000), anti-LC3 (1:1,000), anti-PTEN (1:500), anti-p-AKT ${ }^{\mathrm{S} 473}(1: 1,000)$, anti-AKT $(1: 1,000)$ or anti-GAPDH $(1: 1,000)$ primary antibodies diluted in $5 \%$ BSA solution and incubated at $4^{\circ} \mathrm{C}$ overnight. The membranes were then washed in TBST and incubated with horseradish peroxidase (HRP)-conjugated goat anti-rabbit IgG secondary antibody (1:1,000; cat. no. 1706515; Bio-Rad, Hercules, CA, USA) for $1 \mathrm{~h}$ at room temperature. After washing again in TBST, the membranes were developed using Lumi-Light chemiluminescence substrate (Roche, Indianapolis, IN, USA). Digital imaging and signal quantification were performed on a Chemi-Genius2 Bio-Imager (Syngene, Frederick, MD, USA) using GeneTools software Gene tools 4.01(c) (Syngene).

Statistical analysis. Statistical significances between groups were determined by two-tailed Student's t-test. $\mathrm{P}<0.05$ was considered to indicate a statistically significant difference. 
$\mathbf{A}$
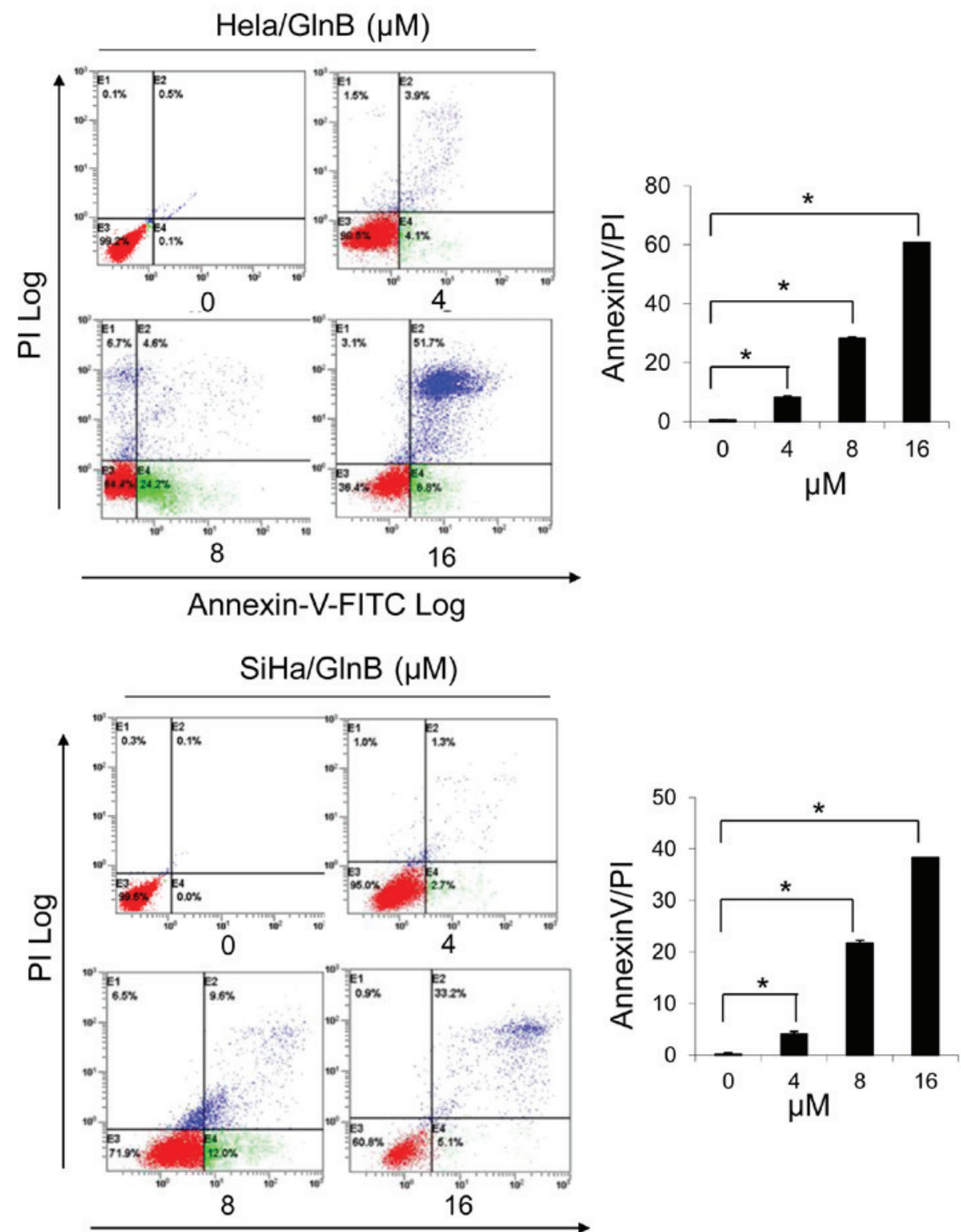

Annexin-V-FITC Log

B
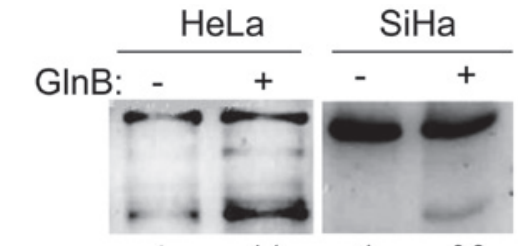

PARP1

Cleaved PARP1

14.
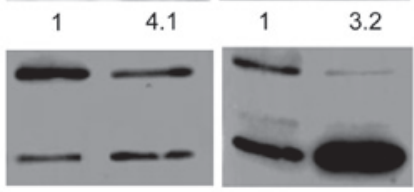

LC3 I

LC3 ॥

LC3 I

LC3 II

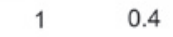

1
1

0.3

2.6
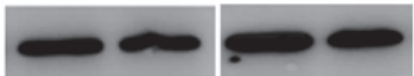

\section{GAPDH}

Figure 2. GlnB induces apoptosis and autophagy in HeLa and SiHa cells. HeLa and SiHa cells were treated with various concentrations of GlnB $(0,4,8$, and $16 \mu \mathrm{M}$ ) for $24 \mathrm{~h}$. (A) Apoptosis was assessed by flow cytometric analysis of annexin V-FITC/PI staining of HeLa and SiHa cell lines. The data shown are representative of two independent experiments ("P $<0.05$ compared with the $0 \mu \mathrm{M}$ treatment group). (B) HeLa cells were treated with $8 \mu \mathrm{M}$ GlnB or DMSO for $24 \mathrm{~h}$, and the SiHa cells were treated with $6 \mu \mathrm{M} \mathrm{GlnB}$ or DMSO for $24 \mathrm{~h}$. The expression levels of PARP1 and LC3II/I were examined by western blot analysis, and quantified by digitization. GAPDH was the internal control. The data shown are representative of two independent experiments. GlnB, glaucocalyxin B; FITC, fluorescein isothiocyanate; PI, propidium iodide; DMSO, dimethyl sulfoxide; PARP, poly (ADP-ribose) polymerase; LC, light chain; GAPDH, glyceraldehyde 3-phosphate dehydrogenase. 


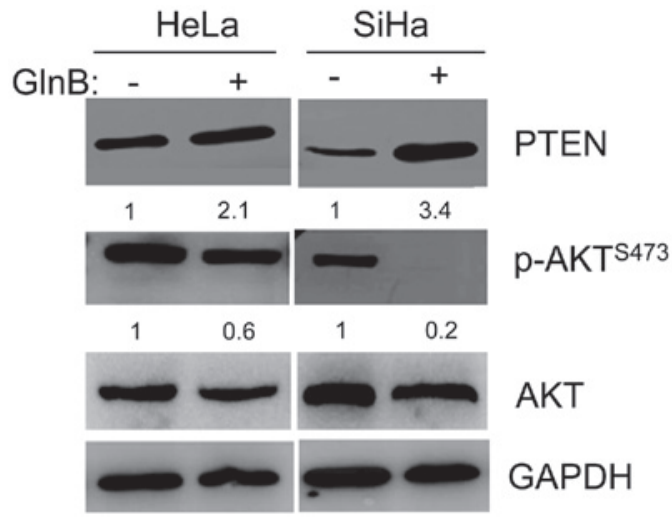

Figure 3. GlnB antitumor activity is involved the PI3K/Akt signaling pathway. HeLa cells were treated with $8 \mu \mathrm{M}$ GlnB or DMSO for $48 \mathrm{~h}$, and $\mathrm{SiHa}$ cells were treated with $6 \mu \mathrm{M}$ GlnB or DMSO for $24 \mathrm{~h}$. Western blot analysis was performed to determine the expression levels of PTEN, Akt and p-Akt (S473) in HeLa and SiHa cells treated with GlnB. The expression levels were quantified by digitization. GAPDH was used as an internal control. The data shown are representative of two independent experiments. GlnB, glaucocalyxin B; PI3K, phosphatidylinositol-4,5-bisphosphate 3-kinase; Akt, protein kinase B; DMSO, dimethyl sulfoxide; PTEN, phosphatase and tensin homolog; p-, phosphorylated; GAPDH, glyceraldehyde 3-phosphate dehydrogenase.

\section{Results and Discussion}

For $>40$ years, natural products have served us well in combating cancer. Of the antitumor compounds used in medicine, three quarters are natural products or their derivatives, including paclitaxel (9).

Ent-kaurane diterpenoids, including GlnA, are known to exhibit strong antitumor activities (5). Compared with GlnA, the only structural difference of GlnB is an acetylated hydroxyl group at $\mathrm{C14}$, and this highly liposoluble acetyl group of GlnB makes the compound easier to penetrate into the cells, thereby exhibiting stronger anti-inflammatory properties (10). The antitumor activity of ent-kaurane diterpenoids is through a similar structure-activity association (5), suggesting that GlnB may have stronger antitumor activity compared with GlnA. Although GlnB has been reported to possess anti-inflammatory activity (10), the role of GlnB in cancer cells remains unclear.

GlnB inhibits cell proliferation in cervical cancer cells in vitro. To examine whether $\mathrm{Gln} B$ affects cervical cancer proliferation, the present study first tested the effect of GlnB on the growth of human HeLa and SiHa cervical cancer cell lines. Following exposure to various concentrations of GlnB for $72 \mathrm{~h}$, cell growth and viability were measured using an MTT assay. GlnB treatment inhibited the growth of $\mathrm{HeLa}$ and $\mathrm{SiHa}$ cells in a dose-dependent manner (Fig. 1). The $\mathrm{IC}_{50}$ of GlnB for HeLa and SiHa cells was 4.61 and $3.11 \mu \mathrm{mol} / 1$, respectively (Fig. 1). These data suggested that GlnB treatment inhibited the proliferation of HeLa and SiHa cells. Additionally, the $\mathrm{IC}_{50}$ determined from in vitro proliferation studies will assist with establishing the doses required for in vivo studies, which will also require the pharmacokinetics data of $\mathrm{GlnB}$ and the desired inhibition in vivo.

GlnB induces apoptosis and autophagy in HeLa and SiHa cells. Yang et al (11) reported that GlnB induces apoptosis in human HL-60 leukemia cells. Apoptosis is a major route to eradicate cancer. Therefore, the present study investigated whether GlnB regulated apoptotic cell death in $\mathrm{HeLa}$ and $\mathrm{SiHa}$ cells. The cells were treated with varying doses of GlnB for $24 \mathrm{~h}$ and apoptosis were assessed using annexin V staining. GlnB treatment increased the population of annexin V/PI-positive cells in a dose-dependent manner in each cell line (Fig. 2A). The expression of the apoptosis marker PARP1 was examined by western blot analysis. As shown in Fig. 2B, the level of activated and cleaved PARP1 was upregulated in HeLa cells treated with $8 \mu \mathrm{M}$ GlnB compared with DMSO-treated control cells. Similar results were observed in the $\mathrm{SiHa}$ cells treated with $6 \mu \mathrm{M}$ GlnB (Fig. 2B). Autophagy is another important mechanism for cancer cell death. Therefore, the effect of GlnB treatment on autophagy was assessed by determining the expression of LC3 II/I by western blot analysis. LC3 I is cleaved to from LC3 II under such conditons. GlnB treatment increased LC3 II/I protein cleavage in both cell lines compared with the DMSO-treated controls (Fig. 2B). These results indicated that GlnB induces autophagy in cervical cells.

Phosphatidylinositol-4,5-bisphosphate 3-kinase $(\mathrm{PI} 3 \mathrm{~K}) /$ protein kinase $\mathrm{B}(\mathrm{Akt})$ is the central pathway in determining the onset and progression of apoptosis and autophagy $(12,13)$. GlnA has been shown to inhibit Akt phosphorylation, suppress proliferation and promote apoptosis in human malignant glioma U87MG cells (7). Therefore, the present study next examined whether PI3K/Akt signaling is involved in GlnB-induced apoptosis and autophagy. The expression of key signaling proteins involved in this pathway, PTEN, Akt and p-Akt ${ }^{\mathrm{S} 473}$, were assessed by western blot analysis. The results showed that GlnB increased the expression of PTEN and decreased the expression of p-Akt ${ }^{\mathrm{S} 473}$ (Fig. 3). The total Akt remained unchanged by GlnB treatment (Fig. 3). These findings indicated that the antitumor activity of GlnB is associated with the PI3K/Akt signaling pathway.

In conclusion, the present results demonstrated that GlnB inhibited the proliferation of human cervical cancer cells in vitro via the induction of apoptosis and autophagy, which may be mediated by the PI3K/Akt signaling pathway. Future studies are required to identify the direct molecular target(s) of GlnB and to evaluate its antitumor effects in vivo using animal models. Collectively, the present study demonstrated that GlnB possesses antitumor properties in cervical cancer and may have a therapeutic potential against this deadly disease.

\section{Acknowledgements}

The present study was supported by the Research Grants of Henan Science and Technology Project (no. 142102310301).

\section{References}

1. Kim WK, Bang MH, Kim ES, Kang NE, Jung KC, Cho HJ and Park JH: Quercetin decreases the expression of ErbB2 and ErbB3 proteins in HT-29 human colon cancer cells. J Nutr Biochem 16: 155-162, 2005.

2. Rose PG, Bundy BN, Watkins EB, Thigpen JT, Deppe G, Maiman MA, Clarke-Pearson DL and Insalaco S: Concurrent cisplatin-based radiotherapy and chemotherapy for locally advanced cervical cancer. N Engl J Med 340: 1144-1153, 1999.

3. Wieringa HW, van der Zee AG, de Vries EG and van Vugt MA: Breaking the DNA damage response to improve cervical cancer treatment. Cancer Treat Rev 42: 30-40, 2015. 
4. Sun Y: The role of chinese medicine in clinical oncology. Chin J Integr Med 20: 3-10, 2014.

5. Sun HD, Huang SX and Han QB: Diterpenoids from isodon species and their biological activities. Nat Prod Rep 23: 673-698, 2006.

6. Gao LW, Zhang J, Yang WH, Wang B and Wang JW: Glaucocalyxin A induces apoptosis in human leukemia HL-60 cells through mitochondria-mediated death pathway. Toxicol In Vitro 25: 51-63, 2011.

7. Xiao X, Cao W, Jiang X, Zhang W, Zhang Y, Liu B, Cheng J, Huang $\mathrm{H}$, Huo $\mathrm{J}$ and Zhang $\mathrm{X}$ : Glaucocalyxin A, a negative Akt regulator, specifically induces apoptosis in human brain glioblastoma U87MG cells. Acta Biochim Biophys Sin (Shanghai) 45: 946-952, 2013.

8. Han M, Li Z, Guo Y, Zhang J and Wang X: A nanoparticulate drug-delivery system for Glaucocalyxin A: Formulation, characterization, increased in vitro and vivo antitumor activity. Drug Deliv: 1-7, 2015.
9. Demain AL and Vaishnav P: Natural products for cancer chemotherapy. Microb Biotechnol 4: 687-699, 2011.

10. Gan P, Zhang L, Chen Y, Zhang Y, Zhang F, Zhou X, Zhang X Gao B, Zhen X, Zhang J and Zheng LT: Anti-inflammatory effects of glaucocalyxin B in microglia cells. J Pharmacol Sci 128: 35-46, 2015.

11. Yang WH, Zhang Z, Sima YH, Zhang J and Wang JW: Glaucocalyxin A and B-induced cell death is related to GSH perturbation in human leukemia HL-60 cells. Anticancer Agents Med Chem 13: 1280-1290, 2013.

12. Kapoor V, Zaharieva MM, Das SN and Berger MR: Erufosine simultaneously induces apoptosis and autophagy by modulating the Akt-mTOR signaling pathway in oral squamous cell carcinoma. Cancer letters 319: 39-48, 2012.

13. Le XF, Mao W, Lu Z, Carter BZ and Bast RC Jr: Dasatinib induces autophagic cell death in human ovarian cancer. Cancer 116: 4980-4990, 2010. 